\title{
A Study of Personalized Shift Strategy based on Styled Driver Modeling
}

\author{
Yun Tang \\ College of Electrical Engineering \\ Zhejiang University \\ Hangzhou, China \\ 21310113@zju.edu.cn
}

Bin Shi

College of Electrical Engineering

Zhejiang University

Hangzhou, China

21310128@zju.edu.cn

Jie $\mathrm{Hu}$

College of Electrical Engineering

Zhejiang University

Hangzhou, China

Israel1987@126.com

\author{
$\mathrm{Li} \mathrm{Xu}$ \\ College of Electrical Engineering \\ Zhejiang University \\ Hangzhou, China \\ xupower@zju.edu.cn
}

\author{
Wuqiang Meng \\ Ford Motor Research \& Engineering (Nanjing) Co., Ltd \\ Nanjing, China \\ wmeng1@ford.com
}

\begin{abstract}
Shift schedule is an important parameter for automatic transmission vehicle calibrations, which directly affects the vehicle's power and fuel economy. This paper tries to establish a system to optimize shift performance based on the styled driver model. First researchers proposed to imitate the real-world drivers' characters based on a locally designed neural network, which divides the driving style into three catagories, aggressive, moderate and mild. Second, the personalized driver models were tested for simulation under the standard speed profile, e.g. FTP-75. Third, the times series of shift and fuel consumption were analyzed under different driving styles. Finally, the shift map for different driving styles are corrected and the test results demonstrate the effectiveness of this method. The corrected shift schedule can effectively reduce fuel consumption and improve shifting comfort, which is useful for Eco-driving.
\end{abstract}

Keywords-Shift Schedule; Fuel Consumption; Driver Modeling; B-Spline; Driving Style;Times of Shift

\section{INTRODUCTION}

Automatic car has become the mainstream of car market for its simple operation and good driving experience. The shift schedule controls the shift time and the shift gear according to driver's intention, vehicle condition and ambient environment (DEV). Shift schedule drives the gear at the best point in order to achieve the optimal performance, such as vehicle's power and fuel economy. Oil resources are increasingly scarce that reducing vehicle fuel consumption is particularly important, especially for the shift processes and which has become a hot topic in recent years. ${ }^{[1]}$

In the real driving condition, driver-vehicle-road is to form a closed loop system, so the fuel consumption is not only related to vehicle itself and road condition but also driving characteristic. ${ }^{[8]}$ Driving style behaviors mainly include the driver's operation on throttle pedal, brake pedal and steering wheel. Even under the same road condition, different styled drivers perform definitely different on the performance of gear shifting process.

As the vehicle condition is very complex during driving and it is nonlinear for the working environment, driver modeling using traditional PID control method has the limitations of low accuracy and poor applicability. The neural network has a strong self-learning ability and provides a convenient method for nonlinear system. There is no need to know the logical relationships between input and output. The model can be established just based on the actual input data and output data. In this paper, driver models were established by neural network, rather than PID controlmethod for simulation test ${ }^{[9]}$.

The driving styles were divided as aggressive, moderate and mild, which were assessed based on human experience derived from the Ford engineers. Generally speaking, aggressive driver is to operate throttle pedal and brake pedal at maximum amplitude and frequency. Mild driver's throttle and brake action is most soft. Moderate driver is somewhere between the two. Researchers collected the real road driving data from Detroit, United States and established the personalized model for each driver, which can reflect the driving style of each driver intuitively. Then the driver model was tested under the standard speed profile (FTP-75). Researchers analyzed the performance of three driving styles under the basic shift map and the effect of different driving styles on vehicle fuel consumption and shift times. Finally, researchers proposed a method to corrected shift map for different driving styles. 


\section{SHIFT SCHEDULE}

Shift schedule currently used in the automatic transmission is mostly two-parameter shift schedule, which decides the gear and shift timing according to throttle position and vehicle speed. The shift map for 6AT automatic transmission is shown in Fig. 1. Solid lines indicate the upshift curves and dotted lines represent the downshift curves. However, the two-parameter shift schedule has many limitations without considering the impact of shift timing for ramps and corners. They may lead to frequent shift and unexpected shift. In order to solve this problem, many cars now have been equipped with angle sensors and road gradient sensors to introduce ramp, turning signal and other information to correct the two-parameter shift schedule ${ }^{[2]}$.

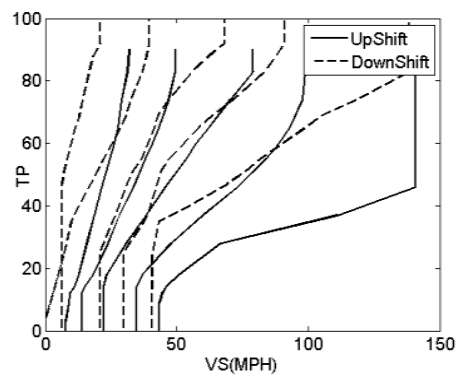

Figure 1. Shift map for 6AT transmission

At same time, two-parameter shift schedule does not consider the effect of different driving styles on the timing of shift. Many cars usually add the sporty driving mode and economy driving mode for diverse gear demand. It cannot solve the problem fundamentally. Because drivervehicle-road is to form a closed loop system, different driving styles will lead to different performance of shift process even if under the same road and vehicle condition. Aggressive drivers usually operate throttle pedal sharply, resulting in frequent shift, the typical process is shown in Fig. 2. It assumes that throttle position of vehicle in the process of driving is unchanged and vehicle is driving at point a ( $4^{\text {th }}$ gear). As a result, the vehicle will accelerate at line $a b$ until to point $b$ ( $4^{\text {th }}$ gear). In this case, if the driver abruptly reduces the throttle, because of inertia, there will be a $4^{\text {th }}-5^{\text {th }}$ upshift when the curve changes to point $\mathrm{c}\left(5^{\text {th }}\right.$ gear). The vehicle speed continues decrease after upshift. If the driver abruptly increases the throttle when the speed drops to point $\mathrm{d}\left(5^{\text {th }}\right.$ gear), the curve will change to point a $\left(4^{\text {th }}\right.$ gear) at line da, leading to a $5^{\text {th }}-4^{\text {th }}$ downshift and causing the rotating shift ${ }^{[3]}$. Rotating shift will be sure to increase the times of shift, followed by the increased fuel consumption and destroy to automotive components.

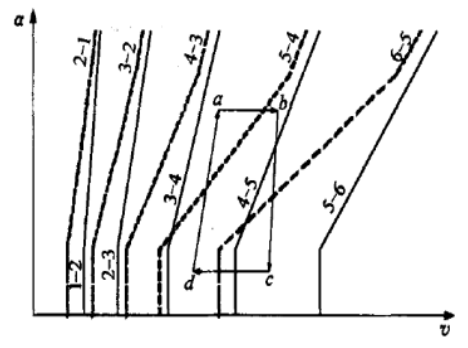

Figure 2. Sketch map of rotating shift
In this paper, researchers propose to introduce the driving style information to achieve two-parameter shift map correction for the purpose to achieve optimal performance, which is shown in Fig. 3.

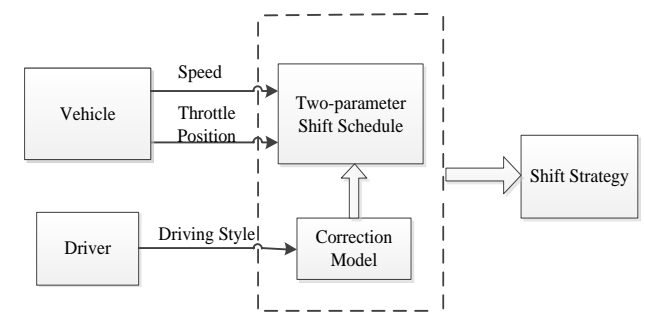

Figure 3. Shift map correction based on driving style

\section{VEHICLE TEST SYSTEM}

Through the whole vehicle structure analysis, the vehicle test system was built shown in Fig. 4, which consisted of the driver model, vehicle model and shift schedule. Neural network has a unique advantage in nonlinear system modeling, with a good self-learning, adaptiveness and nonlinear conversion capacity. While the driver and the vehicle are huge nonlinear systems, the neural network has been used in both driver modeling and vehicle modeling. The vehicle model has integrated the engine model, transmission model and power execution component model.

Using the FTP-75 standard speed profile as the target speed of the closed-loop system, FTP-75 is a standard test condition proposed by the United States Environmental Protection Agency (EPA) for testing exhaust emissions and fuel economy. It simulates a variety of real driving conditions and is widely used in automotive test. The driver model was established using typical local neural network B-Spline. The input is target vehicle speed (FTP75) and current speed (VS) and the output is brake pedal (BP) and throttle position (TP). Shift schedule make the decision of the optimal gear based on the current value of BP and TP. The vehicle model has an effect on the speed after a certain time (VS*) based on the current BP, TP, Gear and VS.

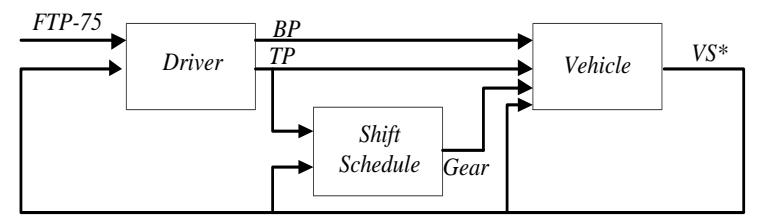

Figure 4. Vehicle test system

\section{A. B-Spline neuralnetwork ${ }^{[4]}$}

B-spline neural network is designed based on the principle of $\mathrm{B}$-spline interpolation function. Its base function consists of a number of local polynomials. Bspline neural network maintains the diversity of sub-spatial information and its principle is shown in Fig. 5. 


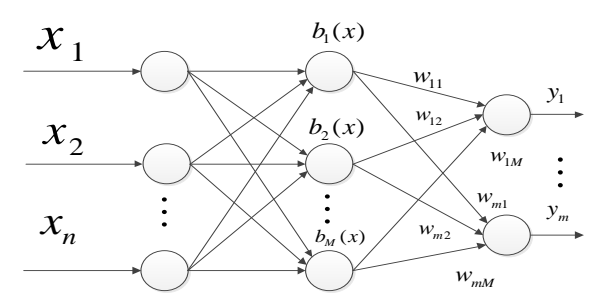

Figure 5. The principle of B-spline neural network

Set the input vector $\mathrm{X}=\left[x_{1}, x_{2}, \ldots, x_{n}\right]^{T}$, then the base function of B-spline neural network is defined as

$$
b_{j_{1} j_{2} \ldots j_{n}}=\prod_{i=1}^{n} N_{i, j_{i}}^{k_{i}}\left(x_{i}\right)
$$

$N_{i, j_{i}}^{k_{i}}\left(x_{i}\right)$ represents the base function of $j_{i}\left(j_{i}=1,2, \cdots, m_{i}\right)$ under the input variable $x_{i}(i=1,2, \cdots, n)$.

The working process of B-spline neural network:

(1)Mapping inputs with base functions: $x \rightarrow b(x)$

Assuming that B-spline with $\mathrm{M}$ hidden units, its base function vector is $b(x)=\left[b_{1}(x), b_{2}(x), \cdots, b_{M}(x)\right]^{T}$

, and $b_{j}(x)$ represents the output value of base function of $j^{\text {th }}$ under the input $x$.

(2)Output of network: $\quad b(x) \rightarrow y$

For B-spline neural network of $\mathrm{n}$ inputs and $\mathrm{m}$ outputs, the $i^{\text {th }}$ output is the product of the corresponding connection weights and the base functions.

$$
y_{i}=\sum_{j=1}^{M} w_{i j} b_{j}(x)=w_{i} b(x), i=1,2, \cdots, m
$$

(3) Weight correction

$$
w_{i j}[k+1]=w_{i j}[k]+\beta\left(y_{i}^{*}-y_{i}[k]\right) b_{i}(x) / b^{T}(x) b(x)
$$

$y_{i}^{*}$ represents the expected output of $y_{i}, y_{i}[k]$ is the actual output at time $\mathrm{k}$, and $\beta$ is the learning rate.

\section{B. Driver Modeling based on B-spline}

Driver model can be expressed as an adaptive MIMO system. When the driver drives the vehicle, he will make adjustment to his driving behavior according to the surrounding environment (different road conditions, weather and other uncontrollable factors). The driver will estimate a target speed based on the ambient environment. The target speed and current speed have an impact on driver's operation on brake pedal (BP) and throttle position (TP).

It is assumed that the vehicle speed is VS[t] at time $t$, at this moment, the brake pedal and throttle position which the driver applied are $\mathrm{BP}[\mathrm{t}]$ and $\mathrm{TP}[\mathrm{t}]$. Because of the automobile inertia and other factors, this operation will affect the speed VS $[\mathrm{t}+\mathrm{k}]$ after $\mathrm{k}$ time period. Let's consider this issue reversely. If the vehicle speed is VS[t] at time $t$ and changes to $\mathrm{VS}[\mathrm{t}+\mathrm{k}]$ after $\mathrm{k}$ time period, it means that there must be $\mathrm{BP}[\mathrm{t}]$ and $\mathrm{TP}[\mathrm{t}]$ at time $\mathrm{t}$. Using the direct inverse modeling shown in Fig. 6 and Vehicle Test
Data(VTD) provided by Ford Motor Company, the Bspline neural network can be trained and corrected repeatedly to obtain accurate driver model with $\mathrm{VS}[\mathrm{t}+\mathrm{k}]$, $\Delta \mathrm{VS}[\mathrm{t}+\mathrm{k}]=\mathrm{VS}[\mathrm{t}+\mathrm{k}]-\mathrm{VS}[\mathrm{t}]$ as input and $\mathrm{BP}[\mathrm{t}], \mathrm{TP}[\mathrm{t}]$ as output.

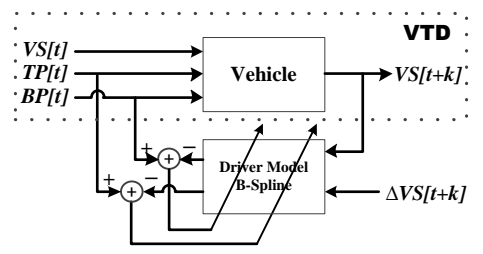

Figure 6. Driver model based on direct reverse modeling method

\section{Vehicle Modeling}

Using the vehicle speed VS[t], the throttle position TP $[t]$, vehicle gear GEA R[t], brake pedal BP $[t]$ at time $t$ as input, the vehicle model output is $\mathrm{VS}^{\prime}[\mathrm{t}+\mathrm{k}]$. Assuming $\mathrm{VS}[\mathrm{t}+\mathrm{k}]$ as the corresponding expected output, VS' $[\mathrm{t}+\mathrm{k}]-$ $\mathrm{VS}[\mathrm{t}+\mathrm{k}]$ is used to correct the vehicle model by error back propagation method. The process keeps cycling until the vehicle model can approximate the actual VTD data. The vehicle model is established as shown in Fig. 7.

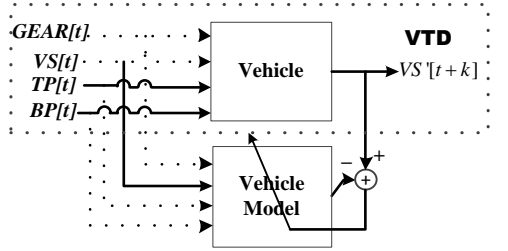

Figure 7. Vehicle model based on neural network

\section{TEST RESULTS}

In order to test the effectiveness and accuracy of the driver model and vehicle model established by neural network, researchers tested aggressive, moderate and mild of each style 6 drivers respectively under FTP-75 standard condition for simulation and analyzed the shift process and performance index. Drivers' driving styles are judged based on human experience obtained by Ford engineers. 16 represent aggressive drivers, 7-12 represent moderate drivers, and 13-18 represent mild drivers.

Brake pedal of different drivers is shown in Fig. 8. The absciss a represents the drivers and the ordinate represents the distribution of every driver's brake pedal over a period of time. From the figure, researchers can see that there are a few large brakes of aggressive drivers but the distribution is not that much. And there is not much difference between moderate drivers and mild drivers. As a result, brake signal apparently cannot distinguish the driving style from drivers effectively.Therefore, researchers adopt the degree of throttle opening to analyze the driver's style and fuel consumption. 


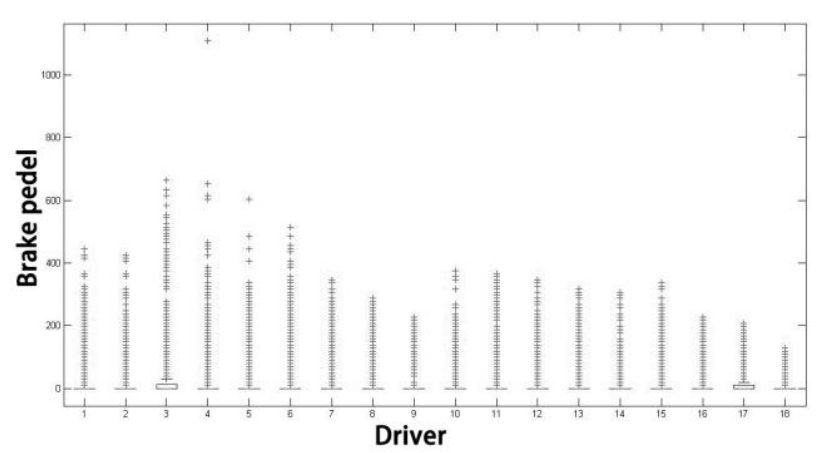

Figure 8. Distribution of different drivers' brake pedal

In order to make styles of different drivers comparable and have enough accuracy in subsequent application, power spectral density index was introduced to quantify the time series of throttle position under FTP-75. Power spectral density describes the energy of signals or system time series changes with frequency. Power spectral density of discrete time series is shown in equation 4.

$$
\phi(w)=\left|\frac{1}{\sqrt{2 \pi}} \sum_{n=-\infty}^{\infty} f_{n} e^{-i w n}\right|^{2}=\frac{F(w) F^{*}(w)}{2 \pi}
$$

Fig. 9 shows the distribution of power spectral density under FTP-75 test. From left to right they represent aggressive, moderate, mild drivers separately. It can be seen from the figure that the throttle position changes violently for aggressive drivers, which is greater than the other two styles. There are clear differences of throttle position output between three styles, providing an effective solution to establish classified index for driving styles.

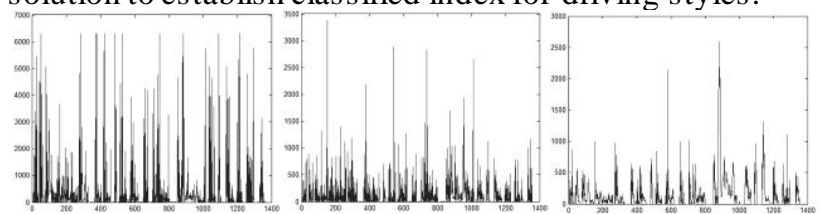

Figure 9. Distribution of power spectral density aggressive, moderate, mild

Other test results are shown in Fig. 10, Fig. 11 and Fig. 12. Fig. 10 represents the aggressive driver. Fig. 11 represents the moderate driver and Fig. 12 is mild driver.

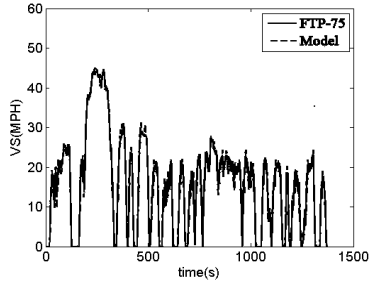

(a)-Speed curve

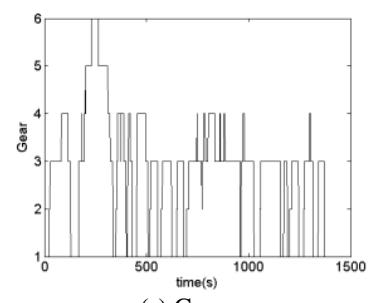

(c)-Gear

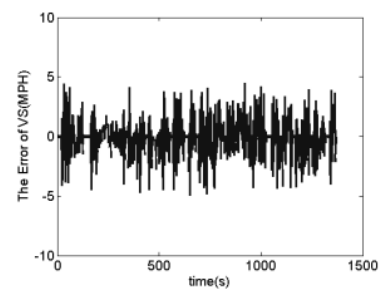

(b)-Speed error

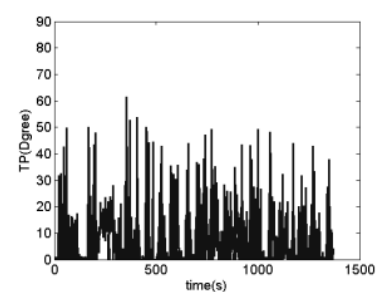

(d)-Throttle position
Figure 10. FTP test results of aggressive driver

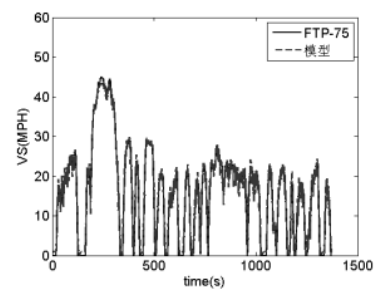

(a)-Speed curve

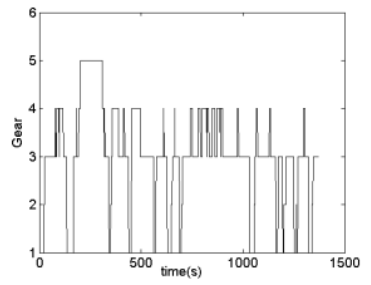

(c)-Gear

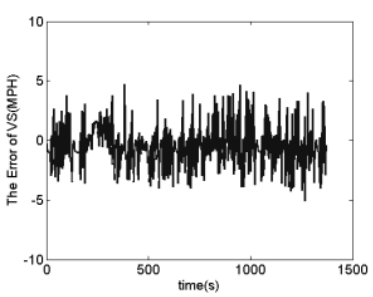

(b)-Speed error

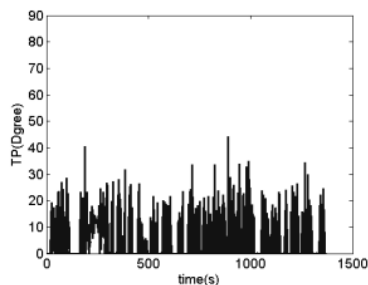

(d)-Throttle position
Figure 11. FTP test results of moderate driver

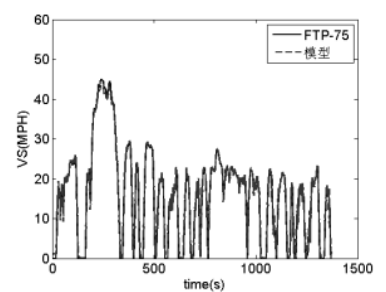

(a)-Speed curve

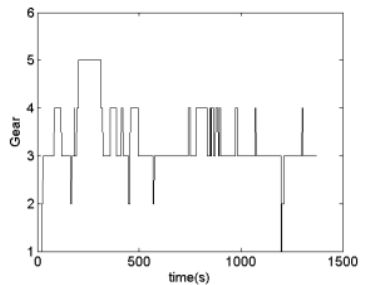

(c)-Gear

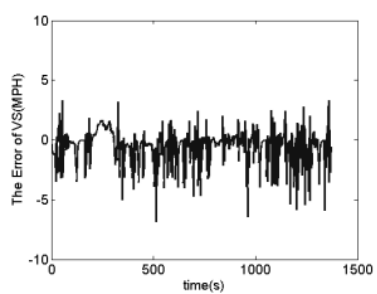

(b)-Speed error

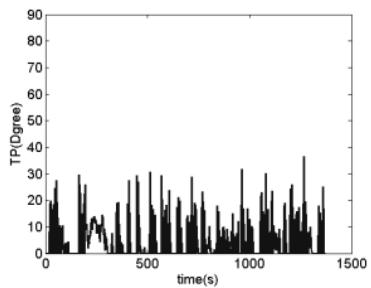

(d)-Throttle position
Figure 12. FTP test results of mild driver

Through the test results, researchers can conclude that drives of every style can control the throttle position to accurately follow the FTP-75 standard speed profile and the following error is relatively s mall. Besides, researchers can see the amplitude and frequency of aggressive driver are biggest and the throttle position has exceeded 50 degree many times. The amplitude and frequency of mild driver is smallest and the throttle position has not been more than 40 degrees. The moderate driver is between them. The results prove that the model is accurate and effective.

Comparing the shift process of three styles drivers, it can be seen aggressive drivers shift most frequently, followed by mild drivers. Mild drivers have a most stable shift position and the gear rarely reduce to $1^{\text {st }}$ gear, ensuring the efficiency of shift. Meanwhile, the aggressive driver is the only one that has reached $6^{\text {th }}$ gear, but then the speed is less than $50 \mathrm{~km} / \mathrm{h}$. This driving process causes higher fuel consumption and allows the engine to produce coke inside. Therefore, the styled drivers will affect not only the times of shift, but also fuel consumption.

There is calculation formula of vehicle fuel consumption, but it is not the key point in this study. In this paper researchers hope to find the relationship between the style and fuel consumption. The throttle position will 
affect the fuel injection quantity after the calculation in ECU. The fuel consumption index is defined as the average degree of throttle position during the time. The fuel consumption index and the times of shift are shown in Table I and Table II. The simulation test results show that the aggressive drivers have maximum fuel consumption and times of shift, followed by the moderate drivers, and the performance of the mild drivers is the best, consistent with the expected results.

TABLE I. FUEL CONSUMPTION INDEX

\begin{tabular}{cccc}
\hline Drives & Aggressive/Degree & Moderate/Degree & Mild/Degree \\
\hline 1 & 6.7700 & 4.6047 & 4.2227 \\
2 & 4.8671 & 5.2175 & 4.2375 \\
3 & 3.7905 & 6.7512 & 3.7710 \\
4 & 8.4489 & 5.0801 & 4.1480 \\
5 & 4.3680 & 5.0774 & 3.7211 \\
6 & 5.0108 & 4.6188 & 3.7756 \\
Average & 5.5426 & 5.2250 & 3.9793 \\
\hline
\end{tabular}

T ABLE II. TIMES OF SHIFT

\begin{tabular}{cccc}
\hline Drivers & Aggressive & Moderate & Mild \\
\hline 1 & 97 & 104 & 35 \\
2 & 105 & 95 & 93 \\
3 & 99 & 89 & 45 \\
4 & 129 & 99 & 101 \\
5 & 107 & 99 & 90 \\
6 & 111 & 105 & 89 \\
Average & 108 & 98.5 & 75.5 \\
\hline
\end{tabular}

\section{SHIFT SCHEDULE CORRECTION}

Busy shift phenomenon is caused by dramatic changing in throttle position. Shift schedule is designed on condition that throttle position changes slowly. It does not apply to dramatic changes in throttle position. In order to avoid the occurrence of cyclic shift, the shift map could be reprocessed based on different driving styles. Researchers can increase the interval between the upshift curves and downshift curves appropriately.

The shift map of mild drivers remains unchanged, while the corrected index of moderate drivers is $\Delta 2$ and aggressive is $\Delta 3$. Correction is simultaneous for upshift and downshift. $\Delta 2$ and $\Delta 3$ are the process of finding the optimal solution which can be produced by particle swarm optimization, genetic algorithms or other tools. In this paper, researchers divided the $10 \%$ range of the standard cures into 6 parts, namely $-10 \%,-6 \%,-2 \%, 2 \%, 6 \%, 10 \%$. Combined with upshift and downshift, there were total 36 cases. Then, researchers modeled and tested another 20 moderate drivers and 20 aggressive drivers under FTP-75 condition. Take the shift map of s mallest fuel consumption as the corrected shift schedule.

In order to test the effectiveness and accuracy of this method, researchers retest the six aggressive drivers and six moderate drives. The simulation test results are shown as Fig. 13 and Fig. 14.

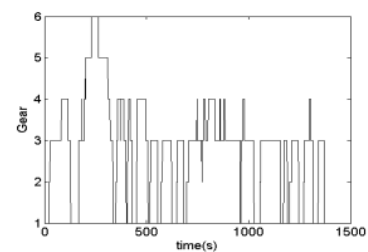

(a)-Gear before correction

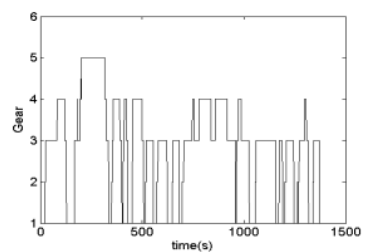

(b)-Gear after correction

Figure 13. Gear of aggressive driver before and after correction

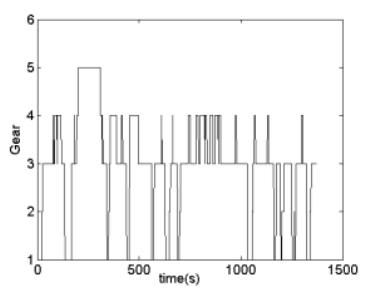

(a)-Gear before correction

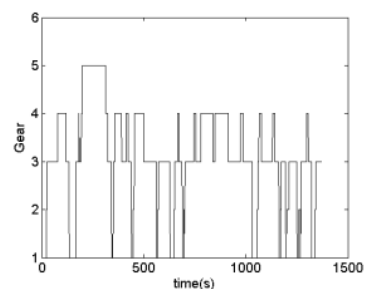

(b)-Gear after correction
Figure 14. Gear of moderate driver before and after correction

Compared with Fig. 13(a) and Fig. 13(b), Fig. 14(a) and Fig. 14(b) respectively, it can be seen that both aggressive drivers and moderate drivers can effectively avoid the rotating shift and reduce the times of shift, especially in time 700s-900s. Thus, it can reduce the wear of vehicle shift actuator and clutch. The fuel consumption index and times of shift are shown in Table III and Table IV.

T ABLE III. FUEL CONSUMPTION INDEX BEFORE AND AFTER CORRECTION

\begin{tabular}{ccccc}
\multicolumn{5}{c}{ Before } \\
After \\
\hline Drivers & $\begin{array}{c}\text { Aggressive/ } \\
\text { Degree }\end{array}$ & $\begin{array}{c}\text { Moderate/ } \\
\text { Degree }\end{array}$ & $\begin{array}{c}\text { Aggressive/ } \\
\text { Degree }\end{array}$ & $\begin{array}{c}\text { Moderate/ } \\
\text { Degree }\end{array}$ \\
\hline 1 & 6.7700 & 4.6047 & 6.7509 & 4.5949 \\
2 & 4.8671 & 5.2175 & 4.8448 & 5.1933 \\
3 & 3.7905 & 6.7512 & 3.7689 & 6.7242 \\
4 & 8.4489 & 5.0801 & 8.4242 & 5.0591 \\
5 & 4.3680 & 5.0774 & 4.3524 & 5.0567 \\
6 & 5.0108 & 4.6188 & 4.9728 & 4.6059 \\
Average & 5.5426 & 5.2250 & 5.5190 & 5.2057 \\
\hline
\end{tabular}

T ABLE IV. T IMES OF SHIFT BEFORE AND AFTER CORRECTION

\begin{tabular}{ccccc}
\multicolumn{5}{c}{ Before } \\
After \\
\hline Drivers & Aggressive & Moderate & Aggressive & Moderate \\
\hline 1 & 97 & 104 & 89 & 88 \\
2 & 105 & 95 & 99 & 81 \\
3 & 99 & 89 & 97 & 75 \\
4 & 129 & 99 & 117 & 87 \\
5 & 107 & 99 & 91 & 87 \\
6 & 111 & 105 & 95 & 91 \\
Average & 108 & 98.5 & 98 & 84.8 \\
\hline
\end{tabular}

The fuel consumption index of moderate drivers and aggressive drivers has decreased obviously from Table III. It can be seen from Table IV that the times of shift have become smaller because of the reducing of busy shift. The simulation test results prove it is effective to correct the shift schedule according to driver's driving style for better performance.

\section{CONCLUSIONS}

In this paper, styled driver model was established by a local neural network and tested under FTP-75 standard condition. Then, researchers analyzed the fuel consumption and shift process of different styled drivers. Finally, researchers proposed a solution to correct shift schedule based on driving style, which have proved to be effective because of its good performance.

Simulation results show that it is possible to provide different shift schedule for different styled drivers to improve and the performance of the vehicle. In the further study, researchers will mainly focus on how to get the corrected shift map more accurately by optimization algorithm. 


\section{References}

[1] Zhou Xuejian, Fu Zhumu, Zhang Wenchun, et al. Research and Development of Shift Schedule of Vehicle Automatic Transmission $[\mathrm{J}]$. Transactions of the Chinese Society for Agricultural Machinery, 2004, 34(3): 139-141.

[2] He Zhongbo, Bai Hongbai, Yang Jianchun, et al. Eliminating Strategy of AMT Vehicles' Shift Hunting [J]. Transactions of the Chinese Society for Agricultural Machinery, 2006, 37(7): 9-13.

[3] Gao Zhenhai, Duan Lifei. Modeling of Driver's Directional Control Behavior with Cerebellar Model Articulation Controller[J]. Aut omotive Engineering, 2012 (4):288-291.

[4] $\mathrm{Xu} \mathrm{Li}$. Intelligent Control and Intelligent System[M]. China Machine Press, 2007.

[5] Ge Anlin. Vehicle automatic transmission theory and design[M]. China Machine Press, 1993:195-220.

[6] Chen Qinghong, Qin Daton,Ye Xin. A study on the three parameter shift schedule of AMT vehicle based on dynamic fuzzy neural net work[J]. Aut omotive Engineering, 2010 (6): 505-509.

[7] Sun Wentao, Chen Huiyan, Wu Chunci. Shifting process adaptive control strategy with electronic automatic transmission [J]. Journal of Mechanical Engineering, 2009, 45(1): 293-299.

[8] Filev D, Lu J, Prakah-Asante K, et al. Real-time driving behavior identification based on driver-in-the-loop vehicle dynamics and control[C]. Systems, Man and Cybernetics, 2009. SMC 2009. IEEE International Conference on. IEEE, 2009: 2020-2025.
[9] Mianzo L, Fricke D, Chabaan R. Road profile control methods for laboratory vehicle road simulators[C]. AUTOTESTCON'98. IEEE Systems Readiness Technology Conference., 1998 IEEE. IEEE, 1998: 222-228.

[10] Lu J, Filev D, Prakah-Asante K, et al. From vehicle stability control to intelligent personal minder: Real-time vehicle handling limit warning and driver style characterization[C]. Computational Intelligence in Vehicles and Vehicular Systems, 2009. CIVVS'09. IEEE Workshop on. IEEE, 2009: 43-50.

[11] Miyajima C, Nishiwaki Y, Ozawa K, et al. Driver modeling based on driving behavior and its evaluation in driver identification[J]. Proceedings of the IEEE, 2007, 95(2): 427-437.

[12] Inata K, Raksincharoensak P, Nagai M. Driver behavior modeling based on database of personal mobility driving in urban area[C]. Control, Automation and Systems, 2008. ICCAS 2008. International Conference on. IEEE, 2008: 2902-2907.

[13] Doshi A, Trivedi M. Examining the impact of driving style on the predictability and responsiveness of the driver: Real-world and Symposium (IV), 2010 IEEE. IEEE, 2010: 232-237.

[14] Zhao G, Wu C. Mathematical modeling of driver speed control with individual differences[J]. Systems, Man, and Cybernetics: Systems, IEEE T ransactions on, 2013, 43(5): 1091-1104.

[15] Abdelwahab H T, Abdel-Aty M A. Development of artificial neural net work models to predict driver injury severity in traffic accidents at signalized intersections[J]. Transportation Research Record: Journal of the Transportation Research Board, 2001, 1746(1):6-13. 